\title{
UN'IPOTESI SU DI UN PERSONAGGIO DI SAMUEL BECKETT
}

\author{
Nota del m.e. AMEDEO BELLINI (*)
}

(Adunanza del 7 febbraio 2019)

SunTO. - Nella commedia di Samuel Beckett En attendant Godot il vero protagonista non è mai in scena, egli è semplicemente l'atteso che non arriva: non sappiamo neppure se è realmente esistente o se è soltanto una fantasia dei coloro che sulla scena dialogano del nulla aspettando colui che sanno non arriverà. Si è discusso molto sul nome Godot: riferimento a Dio indicando vari significati, a personaggi reali incontrati da Beckett e molto altro. Qui si indica un possibile nesso con un personaggio di Honoré de Balzac, un Godeau, anch'esso un assente rievocato che infine, senza apparire in scena, arriva come deus ex machina e salva il protagonista da una difficile situazione. Forse una metafora della contrapposizione tra l'ottimismo provvidenziale della cultura dell'ottocento e lo scetticismo pessimista del novecento.

$$
* * *
$$

ABSTRACT. - In the drama by Samuel Beckett En attendant Godot the real protagonist is never on the stage, he is simply the long-awaited one who never arrives: it is not even known if he really exists or if he is only a fantasy of those who on the stage converse about nothing waiting for who they know will never come. It has been much debated on the name Godot: reference to God suggesting various meanings, reference to real personages met by Beckett and much more. Here it is hypothesized a possible link with a character of Honore de Balzac, a Godeau, he himself an absentee recalled who finally, without appearing on the stage, arrives as deus ex machina and rescues the protagonist from a difficult situation. Perhaps a metaphor for the contrast between the providential optimism of the nineteenth century and the scepticism of the twentieth century.

(") Professore emerito, Politecnico di Milano, Italy.

E-mail: amedeo.bellini@alice.it 
La più famosa opera teatrale di Samuel Beckett, En attendant Godot, venne scritta tra la fine del 1948 ed il gennaio del 1949, in lingua francese, adottata dal 1946 e nella quale egli scriverà la trilogia costituita da Molloy, Malone muore, l'Innominabile, forse le maggiori sue opere; una circostanza non irrilevante per quello che si dirà.

Pubblicato soltanto nel 1952, venne rappresentato per la prima volta nel 1953, con straordinario successo, quando le ferite della guerra si andavano rimarginando, le situazioni di emergenza erano quasi del tutto superate. In Italia alla prima edizione, nello stesso anno dell'originale, ne seguirono altre, numerose ma solo dopo il 1969, ed anche questa data ci suggerisce un collegamento con la situazione di contestazione sociale che esplode in quegli anni (ma ricordiamo che le prime occupazioni di sedi universitarie datano al 1963).

La trama del racconto, nei dettagli, è relativamente poco nota mentre uno dei suoi significati narrativi è divenuto proverbiale, ovviamente soprattutto nella lingua francese: en attendant Godot, ovvero coltivare una speranza che non si realizzerà mai, porre fiducia in qualcosa di irrealistico, coltivare un progetto che non ha fondamento. Il messaggio generalmente avvertito, fra i molti che percorrono la narrazione, è quello pessimistico dell'impotenza umana, dell'angoscia esistenziale, di un divenire che non ha in sé alcunché di provvidenziale, della inevitabilità ed inutilità della sofferenza.

Il protagonista ha la caratteristica di essere fisicamente assente dall'azione scenica ma costante punto di riferimento, forse effettiva esistenza, forse semplice proiezione dell'inquietudine di coloro che sono invece presenti, forse in analogia con l'antitesi tra la fiducia nell'esistenza di Dio o la concezione che si tratti di una pura proiezione di una aspirazione interiore dell'uomo. Attribuire la colpa di una situazione sgradevole ad un fattore esterno è atteggiamento comune sia di una singola persona sia di un gruppo, di una nazione, ma anche, più banalmente, lo è il considerarla tale per l'assenza di qualcuno o per un mancato avvenimento. Ma nel dramma di Beckett ciò che è proprio di una situazione si generalizza come condizione permanente dell'esistenza, come un destino che investe tanto il passato quanto il futuro, senza speranza: vi ci si trova per condizione, si vive vaneggiando nell'auspicio di qualcosa che non accadrà. E se accadesse, vien da dire, sarebbe diverso da quanto ci si attendeva, non necessariamente negativo in sé ma comunque dissonante con le attese. Se una antica tradizione concepisce il destino come un futuro, inconoscibile ed ine- 
vitabile, il fato che può vanificare ogni intenzione individuale, o l'ottimistica fiducia nel progresso "inevitabile" che assorbe ogni individualità, la modernità ha introdotto l'idea che il destino è ciò che la sorte ci ha assegnato senza scelta responsabile, per esempio per nascita, e all'idea di un corso razionale della storia che richiedeva soltanto di essere compreso nelle sue manifestazioni, ha sostituito quella del progetto del futuro, e quindi delle responsabilità. Beckett mette in scena una realtà caratterizzata dall'assenza di storicizzazione del "destino", dei personaggi, solo in minima parte noto, dall'assenza totale di ogni progetto: l'evento atteso che non accade non sembra avere scopo, si attende un Godot che non arriva ma non sappiamo cosa produrrebbe il suo arrivo, ne perché lo si attenda, quale consonanza o dissonanza si produrrebbe fra lui ed i protagonisti fisicamente presenti nella scena: personaggi immobili, secondo alcuni perché imbelli, secondo altri perché consapevoli dell'inutilità di ogni azione, secondo i più perché la condizione esistenziale non lascia loro possibilità alcuna. Una condizione tanto forte da accumunare gli antagonisti, che talora, è stato osservato, sono descritti con fattezze molto simili, con comuni tratta caratteriali; in alcuni casi inizialmente totalmente opposti ma molto meno diversi nel progredire della narrazione per finire infine con l'assomigliarsi.

Si è molto discusso sul nome del protagonista assente: Godot. È opportuno ricordare preliminarmente che Beckett ha spesso rifiutato interpretazioni filosofiche dei suoi personaggi o delle situazioni messe in scena, insistendo invece piuttosto sulla perfezione del meccanismo teatrale, sulle qualità drammatiche e comunicative del testo. E' noto tuttavia che egli era tutt'altro che sprovvisto di conoscenze di filosofia, antica e moderna, ${ }^{1}$ per cui talora si è visto nel suo rifiuto un vezzo narcisistico o una ritrosia dovuta ad una sorta di pudore. A me pare che sia invece naturale che l'autore di teatro, proprio perché non digiuno di conoscenze teoretiche, non desiderasse essere giudicato con una verifica di coerenza sistematica, che avrebbe potuto anche portare ad un giudizio severo.

Le molte ipotesi su di un possibile particolare significato del nome Godot, quali esse siano, non hanno l'avvallo del suo inventore:

1 Esempi in Luigi Ferrante, Beckett. La vita, il pensiero, $i$ testi esemplari, Accademia, Milano 1972, pp. 14-15. 
"Se avessi saputo chi è Godot lo avrei scritto nel copione" ma questo non è che un caso particolare di un atteggiamento generale: Beckett affermava di non volere che si cercassero simboli nella sua opera, si dichiarava un testimone e non un interprete, tantomeno un intellettuale che potesse indicare un atteggiamento di vita. ${ }^{2}$

Frequenti le interpretazioni che attribuiscono un particolare significato al nome Godot: alcune simboliche, rappresenta il destino, ma questa non è soddisfacente a fronte di qualsiasi interpretazione di quel concetto ne sembra che il dramma ne proponga uno proprio; rappresenta la morte, che tuttavia in realtà arriva sempre, ma questo spiegherebbe l'impotenza e la rinuncia a qualsiasi azione dei personaggi che sarebbero di fronte all'ineluttabile. Lo si ritiene riferito a Dio, peraltro citatissimo nel testo assieme a molti riferimenti biblici fino al punto che qualcuno ha avanzato l'ipotesi che il quesito che Beckett realmente pone sia quello che un "... fondamento di senso si manifesti ..." ponendo in definitiva la questione sulla possibilità che Dio si riveli nella storia, ${ }^{3}$ un'ipotesi da tenere in considerazione per quanto si dirà in conclusione. Ancora si sottolinea la manifestazione della sfiducia nell'opera dell'uomo, ma anche nell'opera del Salvatore, che non si manifesta rendendo problematica la grazia salvifica come dono immotivato di Dio, ${ }^{4}$ con nessi con la formazione protestante del giovane Beckett. Altre ipotesi, meno complesse, il riferimento a Godo, che indica Dio nell'irlandese familiare, oppure che si tratti della somma, quasi un lucchetto enigmistico tra Dio e Charlot, ricordando la passione di Beckett per le comiche che lo vedono protagonista ed il senso ironico, surreale, tragicomico della sua drammaturgia. Ancora come sintesi fra i termini "Go" ovvero "vai", e "dot", punto, che indicherebbe un "fermo", metafora della potenziale identità degli

2 Ivi, p. 227: "Beckett non ama essere definito intellettuale perché lo è, evidentemente, e perché si sforza di non esserlo, di trasformare in giuoco, in parodia la sua stessa cultura universitaria”. Si noti che l'aspetto ludico presso alcuni critici è la principale chiave di lettura.

3 Annamaria Cascetta, Il tragico e l'umorismo: studio sulla drammaturgia di Samuel Beckett, Le lettere, Firenze 2000, p. 55 (citato da Federico Platania in http://www.samuelbeckett.it). L'autrice affronta anche i temi della provvidenza ed i suoi rapporti culturali con le concezioni del "progresso", ivi p. 24; per l'indicazione dei riferimenti alla Bibbia in particolare a p. 19

4 Idem, pp. 18, 24 
opposti. ${ }^{5}$ Oppure ancora God, Dio in inglese ed il suffisso francese "ot" per piccolo. Aneddotici i riferimenti: ad un noto ciclista di nome Godeau, all'episodio di Beckett che sale su di un aereo al cui comando è un Godot e dichiara di non fidarsi di un pilota con quel nome; ad una via di Parigi frequentata da prostitute, che tuttavia non risulta sulle attuali mappe della città. L'unica indicazione pervenuta dallo stesso Beckett è che il nome derivi da "godillot", che è uno stivaletto militare, ma si trattava di un suo scherzo. ${ }^{6}$

Anche per gli altri personaggi di questa ed altre commedie si sono fatte ipotesi a proposito di un significato nascosto, di una derivazione dalla volontà dell'autore di suggerire circostanze di fatto, di tempo, relazioni ideali non esplicite, talora per sottolineare la presenza di riferimenti alchemici, psicoanalitici, questi riferibili a Carl Gustav Jung, ma soprattutto di ricondurre annotazioni culturali, riferimenti letterali colti a giochi di parole, spesso enigmistici, ${ }^{7}$ a rompicapo, alla messa in atto di situazioni paradossali, senza via d'uscita.

Questo intrico interpretativo, fra l'accentuazione dell'aspetto ludico ed ironico e di riferimenti colti, i significati impliciti, il rinvio a tesi filosofiche è in realtà senza soluzione perché i due momenti sono compenetrati, si scambiano di ruolo all'interno della commedia e fanno parte essi stessi della messa in scena di opposti che si fondono e diventano simili, dell'assurdità di cercare un ordine, di trovare un filo conduttore unico e da qui il rifiuto di Beckett verso ogni tentativo di esegesi e soprattutto di classificazioni. Non meraviglia quindi che anche chi nega o pone in secondo piano la dimensione comunemente avvertita, l'angoscia dell'attesa e della speranza tradita, paragonabile al significato primario ed immediato dell'opera figurativa evidente ad una prima lettura alla portata di tutti ma che richiede infine ben altri approfondimenti, avverta poi che, "pur dovendosi liberare Beckett dal beckettismo", riconosca che non vi è dubbio che il lettore, o lo spettatore si

5 Si è sottolineato che la tesi potrebbe trovare una conferma in un altro personaggio di Beckett: Rockaby Roc, che significa dondolo, e che sarebbe un suono indistinto, senza significato, ad indicare il nulla, "by", come bay e quindi "addio" saluto di chi parte e quindi in definitiva: mi dondolo, arrivo all'apice, mi fermo, vado via.

6 Molte ipotesi ed aneddoti sono raccolti nella biografia di Samuel Beckett di James Knowlson, Damned to fame: the life of Samuel Beckett, Bloomsbury, London 1996, in italiano, a cura di Gabriele Fresca, Einaudi 2001.

7 Si veda Luigi Ferrante, cit., p. 42. 
trovi di fronte ad una quantità inestricabile di simboli, ${ }^{8}$ pur nel contempo affermando la necessità di non interpretare i contenuti di Godot al di fuori della loro forma ludica, ${ }^{9}$ come tragicommedia sulla condizione dell'attesa. ${ }^{10}$

La sintesi tra divertimento e significati allusivi o posizioni filosofiche trova in Theodor Adorno una perfetta definizione: "Beckett anziché opporre una propria prospettiva filosofica alle altre esistenti, porta alle estreme conseguenze la crisi delle leggi oggettive, immanenti che definiscono i dati, i significati, la comunicazione umana" ... "l'assurdo è reale ... espressione di una cultura totalmente alienata"...". L'assurdo di Camus è una proiezione drammatica dell'esistenza, un documento ideologizzato; l'assurdo di Beckett è la parodia di qualsiasi documento o testimonianza". ${ }^{11}$

Nonostante le interpretazioni "ludiche" si scrive però che in Beckett il novecento è scontato, e riconoscibile "...non è necessario [... ] vivere nella cosiddetta "illusione" dello storicismo per avvertire la negazione del passato e l'assenza del futuro che, paradossalmente, traspare dal neolinguaggio beckettiano". ${ }^{12}$

Cambiamo autore e soffermiamoci su Honoré de Balzac, il prolifico autore de La Commedie bumaine, in particolare su di una azione teatrale brillante, scritta nel 1840 Mercadet le faiseur tradotto in italiano Mercadet l'affarista soltanto nel $1877{ }^{13}$ è stata messa in scena molte volte anche recentemente: qualcuno forse ricorderà la magistrale vulcanica interpretazione di Tino Buazzelli, attore protagonista e regista. Mercadet è un uomo di affari che crea società senza contenuti, vende e compra azioni speculando, utilizzando abitualmente l'aggiotaggio, manovrando al ribasso o al rialzo valendosi anche di persone indotte

8 Luigi Ferrante, cit., p. 9

9 Idem, p. 51.

10 Federico Platania, cit.

11 Citato da Luigi Ferrante, cit., p. 56-

12 Luigi Ferrante, cit. p. 43, 44.

13 Mercadet l'affarista: commedia in 5 atti di Onorato de Balzac, tradotta per le scene dal professore Felice Uda, Libreria editrice, Milano 1877, poi pubblicata da Sonzogno nel 1882. Il termine francese faiseur ha come significato principale quello di fanfarone, sbruffone, spaccone, gradasso, tutti aggettivi che si possono accettare per la descrizione di Mercadet, ed in secondo luogo di affarista, ed anche questo è adeguato. Forse in epoca moderna si sarebbe tradotto con faccendiere. 
a farlo da informazioni opposte da lui ricevute. Mercadet è indebitato fino al collo e sopravvive con espedienti, facendo debiti per dare piccole acconti ai creditori promettendo loro prossimi e facili guadagni; con grande capacità di convinzione strappa denaro a chi chiede la restituzione di quello che ha già dato. Tuttavia la situazione è sempre più critica e riflettendovi con la moglie questa rimpiange e recrimina su di un socio che, in tempi lontani, era scomparso con un bel capitale. Mercadet invece lo usa per promettere ai creditori la riscossione del loro credito con eccellenti utili: il socio non è fuggito, nelle sue parole, ma è nelle Indie dove fa eccellenti affari. A questa trama che serve a Balzac per ironizzare pesantemente su di una certa società legata al mondo dell'economia nelle sue manifestazioni più bassamente speculative, se ne intreccia un'altra, più normale nella letteratura romantica. La figlia di Mercadet ama, riamata, un giovane impiegato con pochi mezzi ma il padre si oppone e prepara un matrimonio con un nobile che ritiene dotato di un buon patrimonio. In realtà costui si è inventato un titolo nobiliare, è ricco solo di debiti, spera di sanare la propria situazione con il denaro di Mercadet. La situazione si svela e l'affarista non ha più motivo di rifiutare la scelta della figlia, tanto più che il futuro genero si scopre proprietario di un piccolo capitale che gli mette a disposizione. Ma la vecchia volpe sfrutta la situazione per un nuovo inganno: chiede all'innamorato della figlia di fingersi il socio tornato in patria, con il suo piccolo capitale darà un nuovo acconto a tutti i creditori per tacitare le loro pretese promettendo il saldo; egli pensa che se si crederà falsa la notizia del suo fallimento gli sarà possibile chiedere altro denaro e tentare nuove speculazioni. Ecco dunque che i creditori si recano nella stanza dove si sarebbe dovuto insediato il futuro genero di Mercadet, per amore suo complice, che però per un contrattempo non è arrivato; tuttavia invece di ricevere un acconto vengono soddisfatti di ogni loro avere. Il socio fedifrago è tornato, si è arricchito, riconosce di averlo fatto con i denari di Mercadet, paga tutti i suoi debiti.

Quel socio si chiama Godeau: la grafia è diversa, ma la pronuncia identica.

Difficile non pensare ad un parallelo tra il Godeau che rappresenta un passato lontano, un riferimento, un ricordo che non sembra avere conseguenze nel presente ma che infine si materializza, con una idea provvidenziale della storia, l'esistenza di una ragione negli avvenimenti che conduce non solo ad un lieto fine come espediente letterario ma 
anche alla una catarsi del protagonista, che decide di lascare gli affari e di ritirarsi in campagna, di assumere un atteggiamento ispirato a principi etici. Mercadet non ha cercato Godeau, ma costui è arrivato, al di là della sua volontà, una presenza che agisce fuori dalla scena, un'assenza che è presenza, che realizza un destino positivo. Vladimir ed Estragon non cercano Godot, non fanno nulla per incontrarlo, non gli vanno incontro, di lui non si sa nulla, soltanto che è atteso ma che non arriva. Un gioco scenico ma certamente un alludere all'angoscia per un'esistenza che appare senza scopo, di cui è ignota la finalità, pervasa da inutili ed inevitabili sofferenze, presentata da uomini che sembra non abbiano interessi di alcun genere, disancorati, senza nessi con una cultura, con un ambiente, entità senza identità, immobili perché consapevoli della loro condizione.

Una coincidenza casuale lo stesso nome nelle due rappresentazioni? quella del cattolico legittimista Balzac così inserita in quella concezione della storia che Nicola Abbagnano definisce "provvidenziale", nella versione idealista come in quella positivista ${ }^{14}$ quella che mette in scena l'angoscia esistenziale del novecento, lo scetticismo che permea molte filosofie del nuovo secolo, la generalizzata rinuncia alla metafisica, l'affermata assenza di una realtà trascendente o la sua indifferenza ai casi umani. Non sembra credibile che Beckett che sceglie il francese per esprimersi, notoriamente conoscitore della letteratura di quel paese, ignorasse la fortunata commedia di Balzac.

Non sono presenti cenni a questa relazione negli scritti che analizzano l'opera di Beckett, ve ne sono alcuni al contrario laddove si parla di Balzac, in genere da parte di chi l'ha messo in scena, ma semplicemente indicando una precedenza, scontata la relazione fra i due nomi, ma senza precisarne la natura. ${ }^{15}$ Una nota più colta, in uno scritto riferito a Balzac, afferma che egli ".. più di cento anni prima di Beckett, con il personaggio di Godeau, aveva messo in scena l'assenza, che non è ancora un vuoto esistenziale" ${ }^{16}$ ciò che però rappresenta la differenza

14 Nicola Abbagnano, Storia, voce in "Dizionario di filosofia", Torino 1964

15 In particolare Maurizio Carra, L'affarista Mercadet di Honoré de Balzac, recensione di uno spettacolo teatrale, 7 aprile 2013, reperita in www,teatrion line.com, 2013-2014 che esplicitamente afferma che al Godeau di Balzac è ispirato il Godot di Beckett, senza altre considerazioni. Il volume di Annamaria Cascetta, estremamente analitico si conclude con indice dei nomi citati: Balzac non vi figura.

16 Anonimo, Le Faiseure (Mercadet) d'Honoré de Balzac, in "Le Cabinet des 
essenziale. Questo concetto è dichiaratamente figlio di una osservazione di Roland Barthes, riferita al Balzac di Mercadet, che, involontariamente, ci porta verso Beckett “... il gran tema del Faccendiere è dunque l'assenza. Questa assenza è incarnata: è Godeau, il socio fantasma che si attende sempre, che non si vede mai, e che finisce per farne la fortuna muovendo dalla sua assenza. Godeau è una invenzione allucinante; Godeau non è una creatura, è un'assenza ma questa assenza esiste, perché Godeau è una funzione: tutto il nuovo mondo è forse in questo passaggio dall'essere all'atto, dall'oggetto alla funzione; non c'è più bisogno che le cose esistano, basta che funzionino; o piuttosto che esse possano funzionare senza esistere. Balzac ha visto la modernità che si annunciava, non più come il mondo dei beni e delle persone (categorie del codice Napoleonico) ma come quello delle funzioni e dei valori: ciò che esiste, non è più ciò che è, è ciò che sta. Nel Le Faiseur, tutti i personaggi sono vuoti (salvo le donne) ed esistono perché, precisamente, il loro vuoto è contiguo: essi si giustificano l'uno con l'altro". ${ }^{17}$ Nessun collegamento con l'opera di Beckett, che sembra essere la naturale conseguenza di quanto scrive Barthes: è nell'Irlandese che il tema dell'assenza diviene centrale, e con essa l'attesa, inutile e vuota di contenuto, ma proprio per questo caratterizzante l'esistenza dei protagonisti di En attendant Godot.

Una possibile relazione tra Godot e Godeau che non pretende di sostituire le altre individuate o supposte dalla critica (ma siamo sicuri che siano due cose diverse?).

Possibile anche che Beckett non ci abbia mai pensato, ma qui mi si consenta, con consapevolezza della grande differenza tra i due casi, di citare Giulio Carlo Argan, autore di un eccezionale saggio su Walter Gropius e la sua scuola di architettura, ${ }^{18}$ punto di riferimento imprescindibile di ogni studio successivo. In un incontro il maestro del razio-

Antiques - La bibliothèque électronique du Québec”, pubblicato il 16 dicembre 2016.

17 Roland Barthes, «Vouloir nous brûle ...>, in "Essai critiques", Editions du soleil, Paris 1964, pp. 90-93, il saggio è datato 1957); in italiano Einaudi, Torino 1972, pp. 44-48. Il titolo è parte di una citazione. "Vouloir nous brûle et pouvoir nous détruit: mai savoir laisse notre faible organization dans un perpétuele état de calme ", che, non specificato da Barthes, è di Honoré de Balzac. La peau du chagrin, Gosseline, Parigi 1833.

18 Giulio Carlo Argan, Walter Gropius e la Baubaus, Einaudi, Torino 1957. 
nalismo architettonico tedesco osservò che il critico gli aveva fatto dire cose che non aveva mai pensato. Argan, forse dimenticando di essersi allontanato dall'idealismo, rispose che l'importante stava nel fatto che Gropius le avesse fatte pensare a lui. ${ }^{19}$

19 Ricordo personale di una intervista a Giulio Carlo Argan, pubblicata in “TeMA", 1, 1993, Angeli, Milano 1993, pp. 57-64, dove l'episodio non è riportato; nessuna polemica nel ricordo di Argan che aveva con Gropius un rapporto di sincera amicizia. Un'ulteriore nota personale: avendo letto Balzac molto prima di Beckett quando con questi mi sono incontrato il rapporto tra Godeau e Godot mi è sempre apparso del tutto ovvio; varie letture sulla discussione di un possibile significato allusivo in Beckett sono state accolte come un'aggiunta ad un dato che in verità risultava poi essere un'assenza. Assenza anche nel Trèsor de la langue Français, dictionnaire de la la langue du XIX et du XX siècle, pubblicato dal Centre national de la recherche scientifique, Centre de recherche pour un Trésor de la langue Français, tomo terzo, Nancy 1974: l'illustrazione del verbo "attendre" comporta la citazione di 41 esempi letterari di 32 autori diversi, ma Beckett manca; il testo di François Etienne, Dizionario dei modi di dire francese-italiano, italiano-francese, Hoepli, Milano 1991, non riporta en attendant Godot. 